\title{
The Response of Human Thermal Perception and Skin Temperature to Step-Changed Activity Level
}

\author{
Diyi Tan, Hong Liu, and Yuxin Wu
}

\begin{abstract}
Considerable researches have been conducted on human thermal comfort under steady state while studies on human thermal perception under non-steady-state is not sufficient. This paper investigates thermal perception of people who alternated between sedentary exercise and walking on a treadmill in $20^{\circ} \mathrm{C}$ and $25^{\circ} \mathrm{C}$. Experiments in a well-controlled climate chamber, including both physiological measurements and subjective questionnaires, were conducted. The procedure of the experiments was as follows: subject sat for $30 \mathrm{~min}$ in climate chamber to calm down before entered into stage of activity level step changing, which comprised 30min of walking at $0.9 \mathrm{~m} / \mathrm{s}$ followed by $30 \mathrm{~min}$ of sitting and then walking at $1.2 \mathrm{~m} / \mathrm{s}$ followed by $30 \mathrm{~min}$ of sitting. 20 subjects were involved. The results indicate that there is poor correlation between thermal sensation vote and skin temperature in activity level transient periods, and the PMV is not suitable to predict the thermal sensation of people occupying dynamic activity when their thermal sensation does not achieve to steady state. However, there is approximately linear correlation between thermal sensation vote and variation rate of temperature with time in the first few minutes after subjects stopped walking.
\end{abstract}

Index Terms-Dynamic thermal comfort, activity level, physiological response.

\section{NOMENCLATURE}

PMV: Predicted Mean Vote

TSV: Thermal Sensation Vote

TCV: Thermal Comfort Vote

Ta: Indoor air temperature, oC

MRT: Mean radiant temperature

$\mathrm{v}$ : Air velocity, $\mathrm{m} / \mathrm{s}$

RH: Relative humidity, \%

\section{INTRODUCTION}

To date, many researches have been done on human thermal comfort in buildings, based on which many mathematical models of human body thermal regulation have been developed. One of the most popular index among the models based on Fanger's human body heat balance theory [1], PMV, has been adopted by many nationals and international standards, including ISO 7730 [2], ASHRAE 55 [3] and Chinese GB/T 50785 [4], etc. In Fanger's theory, $\mathrm{PMV}$ can be calculated through the following function.

$$
\mathrm{PMV}=f\left(\mathrm{M}, \mathrm{I}, \mathrm{T}_{\mathrm{a}}, \mathrm{T}_{\mathrm{r}}, \mathrm{RH}, \mathrm{v}\right)
$$

Manuscript received November 23, 2016; revised February 3, 2017.

The authors are with the Chongqing University, Chongqing, China (e-mail: tandy017@outlook.com). where $M$ is metabolic rate $\left(\mathrm{W} / \mathrm{m}^{2}\right), \mathrm{I}$ is clothing insulation(clo), Ta is air temperature(oC), Tr is radiant temperature(oC), $\mathrm{RH}$ is relative humidity $(\%)$, and $\mathrm{v}$ is air velocity $(\mathrm{m} / \mathrm{s})$.

Scholars in China developed considerable researched on thermal response of people under steady state in typical thermal environment [5]-[7]. While research on thermal perception of people under relative high level of activity or dynamic state of activity is comparatively less, such as people in store building, entertainment places and ticket-hall, etc. According to the steady model of thermal comfort, the metabolic rate will in-crease 0.3 met when people change their activity level from sitting to walking, which means changing $2.4 \mathrm{oC}$ of the human preferable temperature [8]. In the experiment of Gagge, et al. four healthy male subjects dressed in shorts, pedalled a bicycle ergometer $(50 \mathrm{rpm})$ at $25 \% .50 \%$ and $75 \%$ maximal oxygen uptake and at 10,20 and 30 ambient air temperature (RH 40\%) and it took 20 30mins for their thermal comfort and sensation to arrive new steady state. In his conclusion, the judgement of temperature is dominated by the initial rise of the mean body temperature in the initial transient period while related to sensor mechanisms in skin after the initial transient passed [9]. In the experiment of Nielsen and Endrusick, eight male sub-jects were asked to do a twice-repeated bout of 40-mincycle exercise followed by $20 \mathrm{~min}$ of rest. The conclusion of their research is that the various sensations of temperature correlated best with core temperature [10]. T. Goto et al. proposed a model to estimate transient thermal sensation after metabolic step-change, based on which weighting factors were suggested for prediction of transient thermal sensation. Rowe collected a total of 1627 activity reports with current reports of clothing worn and measurement of physical variables and found that more recent activities would be more influential than earlier ones on the current thermal sensation [11]. In the former studies, the sample size of laboratory experiments was not sufficient and the influence of metabolic transient on thermal sensation and thermal comfort was not quantified except the research of T. Goto. This study aims to explore thermal perception of people occupying dynamic level of activity. And ask the question whether it is possible to quantify the predicted thermal comfort of people who alternated between sedentary exercise and walking on a treadmill.

\section{METHOD}

\section{A. Experimental Protocol}

The experiment was carried out in a climate chamber $(\mathrm{L} * \mathrm{~W} * \mathrm{H}=4 \mathrm{~m} * 3 \mathrm{~m} * 3 \mathrm{~m})$, in which a treadmill and a chair were placed to simulate the station of walking and sitting. The 
layout plan of climate chamber was shown in Fig. 1. The room outside the climate chamber is a precisely controlled environment, which served as a transitional place to reduce the interference of outdoor environment. The environmental parameters of climate chamber are controlled by refrigerating unit, air handler, humidifier and converter to control air velocity. The control range and precision of climate chamber are presented in Table I.

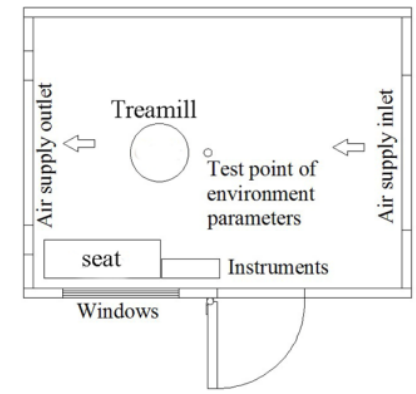

Fig. 1. The layout plan of climate chamber.

TABLE I: The CONTROL RANGE AND PRECISION OF CLIMATE CHAMBER

\begin{tabular}{llll}
\hline \hline & $\begin{array}{l}\text { Environment } \\
\text { parameter }\end{array}$ & Range & Precision \\
\hline Climate & Dry-bulb & $-5 \sim 40^{\circ} \mathrm{C}$ & $\pm 0.3^{\circ} \mathrm{C}$ \\
chamber & temperature & $10 \% \sim 90 \%$ & $\pm 5 \%$ \\
& Relative humidity & $0.1 \sim 2 \mathrm{~m} / \mathrm{s}$ & $0.3 \mathrm{~m} / \mathrm{s}$ \\
& Air velocity & -5 to $60^{\circ} \mathrm{C}$ & $0.10 \mathrm{C}\left(0-20^{\circ} \mathrm{C}\right)$ \\
& Air temperature & & $0.133^{\circ} \mathrm{C}\left(20-40^{\circ} \mathrm{C}\right)$ \\
& & & $0.05 \mathrm{~m} / \mathrm{s}(0-0.5 \mathrm{~m} / \mathrm{s})$ \\
LSI & Air velocity & $0.01 \sim 20 \mathrm{~m} / \mathrm{s}$ & $0.1 \mathrm{~m} / \mathrm{s}(0.5-1.5 \mathrm{~m} / \mathrm{s})$ \\
& & & $4 \%(>1.5 \mathrm{~m} / \mathrm{s})$ \\
& Relative humidity & $0 \sim 100 \%$ & $\pm 2 \%$ \\
& Black-bulb & -40 to $80^{\circ} \mathrm{C}$ & \\
& temperature & & \\
\hline \hline
\end{tabular}

Two different background temperatures were set in the climate chamber, $20^{\circ} \mathrm{C}$ representing slightly cool condition and $25^{\circ} \mathrm{C}$ representing neutral condition. The humidity of climate chamber was set at $50 \%$ and air velocity was $0.1 \mathrm{~m} / \mathrm{s}$ in both case. The temperature in the transitional room was set at the same temperature as that in the climate chamber. Fig. 2 shows the detailed experiment protocol. Subjects change clothes, learn the procedure of experiment and wear physiological sensors in the transitional room, and take $30 \mathrm{~min}$ to reach a stable condition. Then they moved to the climate chamber of cool condition $\left(20^{\circ} \mathrm{C}\right)$ or neutral condition $\left(25^{\circ} \mathrm{C}\right)$ for $150 \mathrm{~min}$. In the walking period, subjects were required to walk at a certain speed on the treadmill, as shown in Fig. 3.

To better set the environmental condition, PMV of each period in both cases were calculated in advance basing on the reference value in ASHRAE 55, in which $\mathrm{M}$ is 1.0 met when sitting, 2.0 met when walking at $0.9 \mathrm{~m} / \mathrm{s}$ and 2.6 met when walking at $1.2 \mathrm{~m} / \mathrm{s}$. The result of calculation is listed in Table II. The whole experiments were completed in Chongqing at April and early May in 2013, during this period, the outdoor temperatures ranged from $19^{\circ} \mathrm{C}$ to $33^{\circ} \mathrm{C}$.

\section{B. Environmental Measurement}

The environmental parameters which influencing human thermal comfort include air temperature, air velocity, air humidity and black-bulb temperature. Thermal Comfort Monitoring Station Equipment (LSI, Italy, Fig. 4) was adopted to monitor the above four parameters of climate chamber. The measuring range and precision of LSI is listed in Table I.

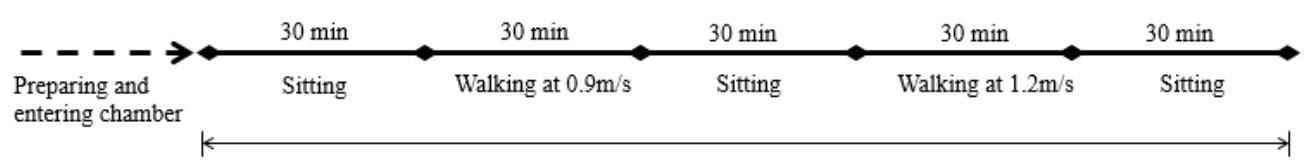

vote at $0,1,2,3,4,5,7,10,15,20,25 \mathrm{~min}$ in each $30 \mathrm{~min}$ of recording period, measuring skin temperature during the whole experiment

Fig. 2. Experimental protocol.

TABLE II: PMV OF EACH EXPERIMENTAL PERIOD IN BOTH CASES

\begin{tabular}{|c|c|c|c|c|c|}
\hline \multirow[b]{2}{*}{ Case } & \multicolumn{2}{|c|}{ Clothing insulation } & \multicolumn{3}{|l|}{ PMV } \\
\hline & Sitting stage & Walking stage & Sitting stage & $\begin{array}{l}\text { Walking stage } \\
(0.9 \mathrm{~m} / \mathrm{s})\end{array}$ & $\begin{array}{l}\text { Walking stage } \\
(1.2 \mathrm{~m} / \mathrm{s})\end{array}$ \\
\hline Slightly cool & 0.85 & 0.7 & -1.2 & 0.4 & 1.0 \\
\hline Neutral & 0.85 & 0.7 & 0 & 1.2 & 1.8 \\
\hline
\end{tabular}

TABLE III: ANTHROPOMETRIC DATA OF SUBJECTS (MEAN \pm SEM)

\begin{tabular}{llllll}
\hline \hline Sex & Number $(\mathrm{n})$ & Age $($ year $)$ & Height $(\mathrm{cm})$ & Weight $(\mathrm{kg})$ & Body $\operatorname{area}\left(\mathrm{m}^{2}\right)$ \\
\hline Male & 10 & $23 \pm 0.4$ & $174.4 \pm 3.5$ & $63.5 \pm 3.7$ & $1.76 \pm 0.06$ \\
Female & 10 & $23.2 \pm 0.7$ & $162.9 \pm 5$ & $52.1 \pm 4.2$ & $1.54 \pm 0.08$ \\
Total & 20 & $23.1 \pm 0.6$ & $168.7 \pm 7.2$ & $57.8 \pm 6.9$ & $1.65 \pm 0.13$ \\
\hline \hline
\end{tabular}

\section{Participants}

Table III summarizes subjects' profiles. 20 healthy college students were involved in both two environ-mental cases. In order to ensure the validity of experimental data, all the subjects were asked to avoid caffeine, alcohol, smoking, and strenuous activities, and to have a good sleep at the night before experiment. Each subject was familiar with experimental procedures. During the experiment, they were required to wear a uniform clothing including long-sleeved T-shirt, long-sleeved, long cotton trousers, cotton socks and sneakers with an insulation level of 0.7 clo referenced from clothing garment checklists in ASHRAE standard 55[3]. It is needed to append the thermal insulation of chair, the adjunctive insulation level of which is 0.15 clo. During the experiment, the subjects cannot leave from the climate chamber and were forbidden to eat, but they were allowed to drink tepid water. 


\section{Physiological and Measurements}

Half experiment time, subjects were in the motion state and copper-constantan thermocouples cannot stick on the skin of subjects and even drop off. However, the object of the research only needs we record the variation of skin temperature when subjects in dynamic motion state so the high-accuracy method of measuring skin temperature is not that necessary. Therefore, the Sense Wear Armband was adopted to measure subjects' skin temperature. The measuring range of Sense Wear Armband is 20 40oC, the accuracy is $\pm 0.2 \mathrm{oC}$ and the response time is $0.2 \mathrm{~s}$

The subjective questionnaire is recorded for every recording period of $0.5 \mathrm{~h}$. In the $0 \mathrm{~min}, 1 \mathrm{~min}, 2 \mathrm{~min}, 3 \mathrm{~min}$, $4 \mathrm{~min}$, 5min, $7 \mathrm{~min}$, 10min, 15min, 20min, $25 \mathrm{~min}$ of a recording period, subjects were asked to vote their thermal sensation, thermal comfort, and sweating rate, using the evaluation scales in Table IV.

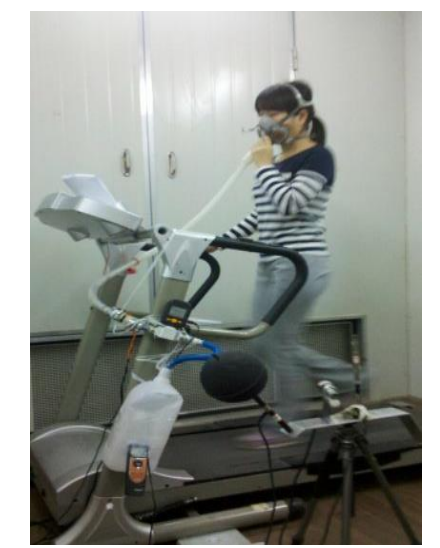

Fig. 3. Subjects walking on the treadmill.

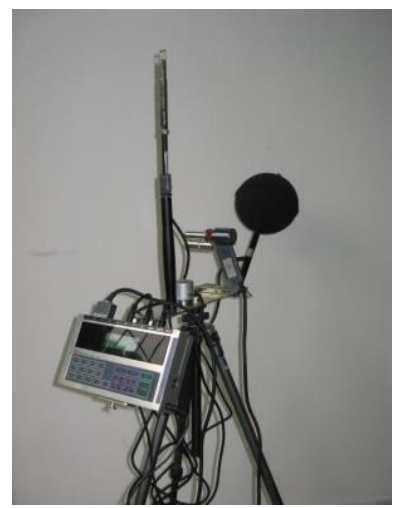

Fig. 4. Experimental apparatus, LSI

TABLE IV: INDEX SCALE OF SELF-EVALUATION

\begin{tabular}{llll}
\hline \hline Scale & TSV & TCV & Sweating \\
\hline 3 & Hot & Very uncomfortable & Heavily sweating \\
2 & Warm & Comfortable & Sweating \\
1 & Slightly warm & Just uncomfortable & Slightly sweating \\
0 & Neutral & Comfortable & None \\
-1 & Slightly cool & - & \\
-2 & Cool & - & \\
-3 & Cold & - & \\
\hline \hline
\end{tabular}

TABLE V: THE MONITORING RESUlt In CLIMATE CHAMBER

\begin{tabular}{llllll}
\hline \hline Temperature case & $20^{\circ} \mathrm{C}$ & & & $25^{\circ} \mathrm{C}$ & \\
\cline { 2 - 3 } \cline { 5 - 6 } & Mean & Std. & & Mean & Std. \\
$\mathrm{Ta}\left({ }^{\circ} \mathrm{C}\right)$ & 20 & 0.06 & & 24.99 & 0.06 \\
$\mathrm{MRT}\left({ }^{\circ} \mathrm{C}\right)$ & 20.13 & 0.06 & & 24.90 & 0.14 \\
$\mathrm{v}(\mathrm{m} / \mathrm{s})$ & 0.10 & 0.01 & & 0.11 & 0.01 \\
$\mathrm{RH}(\%)$ & 52.7 & 1.6 & & 53.4 & 1.3 \\
\hline
\end{tabular}

\section{RESUlTS: THERMAL CONDITIONS}

The monitoring parameters in actual experimental conditions are listed in Table $\mathrm{V}$, from which it can be seen that all the thermal conditions were effectively controlled in accordance with the designed set-points.

\section{RESUlts: SubJECTIVE CONDITIONS}

\section{A. Thermal Sensation Vote}

The TSV shown in Fig. 5. represents the mean thermal sensation vote of all the subjects for both experimental cases. The TSV increased or decreased gradually to a steady state rather than achieve to the value of PMV for the corresponding state immediately when subjects changed their activity level. Therefore, the PMV is not suitable to predict the thermal sensation of people occupying dynamic activity when their thermal sensation does not achieve to steady state.

As shown in Fig. 6, the result of the neutral condition indicate that thermal sensation was more sensitive to and changed faster with down-steps of activity than with up-steps, which is in accordance with the research of T. Goto [11] and the research of de Dear who observed that humans were generally more sensitive to warm-to-cold temperature step changes than they were to cold-to-warm step-changes of equal magnitude [12]. In the study of de Dear et al, the thermal perception was mediated by the ambient environment transient whereas in the current study thermal perceptions were registered by core thermoreceptors rather than peripheral. However, the variation of TSV in cold condition was more sensitive to up-steps of activity, which was opposite to the variation of TSV in neutral condition. It seems that subjects felt warm immediately after they began to walk.

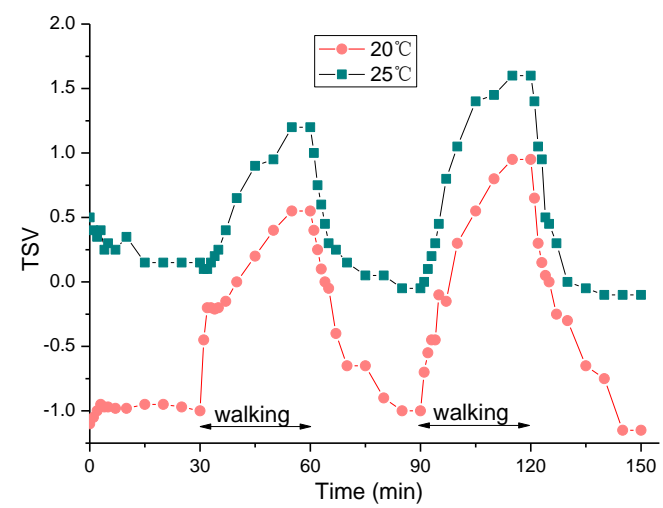

Fig. 5. Changes in overall thermal sensation with time.

\section{B. Thermal Comfort Vote}

As presented in Fig. 7, subjects in sedentary state felt uncomfortable because of cold sensation in the slightly cool environment, this uncomfortable sensation could be relieved rapidly when subjects began walking. While after subjects stopped walking, the uncomfortable sensation increased gradually due to the continued human heat production caused by slow response of human thermal regulation system. While in the neutral condition, subjects felt comfort when sitting and felt uncomfortable when walking due to increasing core temperature. 


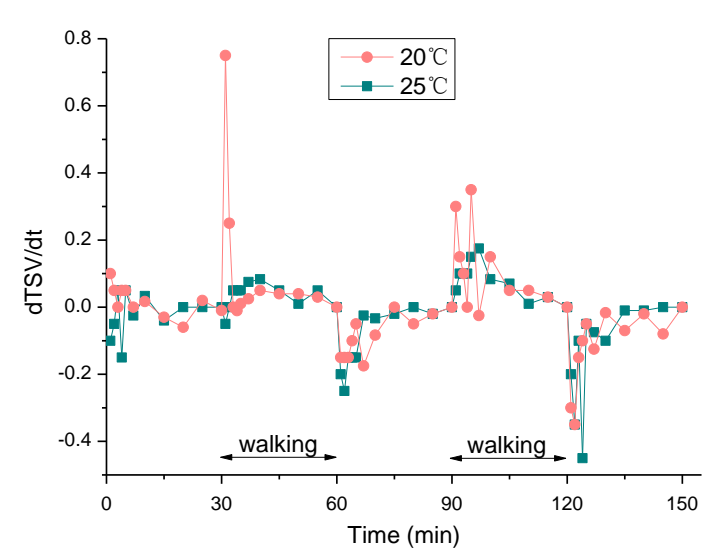

Fig. 6. TSV variation rate with time.

The SI unit for magnetic field strength $H$ is $\mathrm{A} / \mathrm{m}$. However, if you wish to use units of $\mathrm{T}$, either refer to magnetic flux density $B$ or magnetic field strength symbolized as $\mu_{0} H$. Use the center dot to separate compound units, e.g., "A $\mathrm{m}^{2}$."

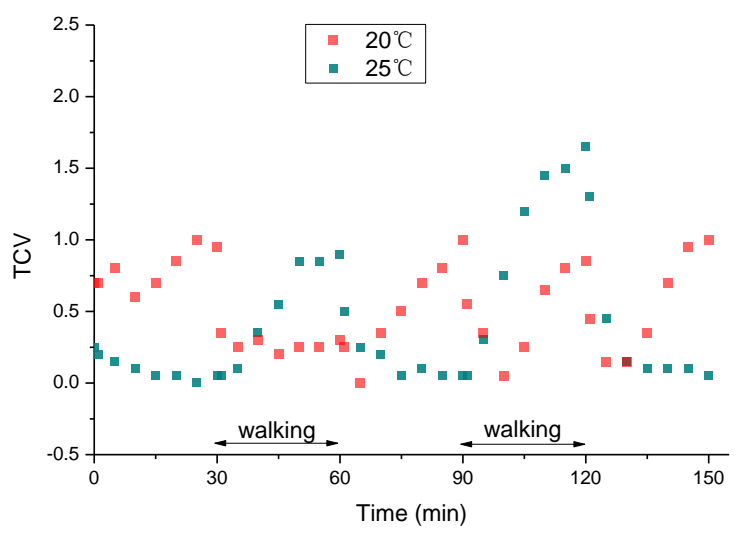

Fig. 7. Changes in overall thermal comfort with time.

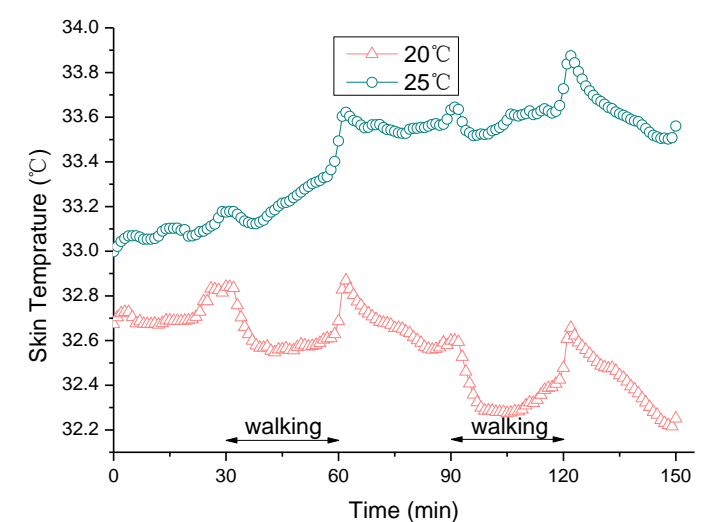

Fig. 8. Changes of skin temperature over time in response to dynamic activity level.

\section{SKIN TEMPERATURE}

It should be noted that the purpose of the present study is to explore the human thermal subjective and physiological responses to dynamic activity level. Therefore, we mainly focus on variation trend of the skin temperature, rather than the accurate value of mean skin temperature. Although the skin temperature measured by Sense Wear Armband cannot rep-resent the body mean skin temperature, the general change is reliable.

The human skin temperature is determined by the combined effect of surrounding environment and their temperature regulation system. When human metabolic rate increases, core temperature increases correspondingly and more heat dissipating is needed. Then the rate of blood flow and perspiration evaporation is accelerated to release more heat to environment under the control of temperature regulation system. Fig. 8 depicts the skin temperature changing with time in both cases. The skin temperature is lower in slightly cool condition than that in neutral condition to reduce heat transfer between human body and environment, which is reconfirmed by Fig. 8. The air layer around the human body is disturbed when body is in motion state, which resulted in strengthened convective heat transfer between the human body and the surrounding air. And the response of human heat production to metabolic rate changing is slower than that of skin temperature to environment. Under the comprehensive action of these factors, the skin temperature decreased in the first few minutes of walking period. When human heat production increases until it can contract the heat dissipating due to convective transfer, the skin temperature will return to a rather steady stage. The skin temperature in the slightly cool condition showed a general decrease trend. This can be explained by that the heat dissipating due to convective heat transfer exceeded the heat production in the slightly cool condition.

The convective heat transfer decreases when people enter in to steady state, while the heat production continue to increase due to its slow response reaction to metabolic rate changing. Therefore, the skin temperature had a sharply increase in the first few minutes of sitting period, which was followed by rather mild state of skin temperature due to heat production decreased.

\section{DYNAMIC RELATIONSHIPS BETWEEN TSV AND HUMAN PHYSIOLOGICAL PARAMETER}

\section{A. Dynamic Thermal Sensation and Skin Temperature}

Fig. 9 plots dynamic thermal sensation against skin temperature for different activity level in both cases. A poor correlation was found between thermal sensation and skin temperature in each case. This is different from the figures under steady and uniform environment, where thermal sensation has a strong linear relationship, or index relationship with mean skin temperature [13]. The reason may be that skin temperature decreased due to perspiration evaporating while the body core temperature in-creased continuously. Therefore, mean skin temperature is not enough to determine the comfort level of occupants when they are occupying in dynamic activity.

\section{B. Dynamic Thermal Sensation and the Variation rate of Mean Skin Temperature}

It can be seen from Fig. 9 that there is a rather significant linear relationship $\left(\mathrm{R}^{2}=0.54,25^{\circ} \mathrm{C}, \mathrm{R}^{2}=0.79,20^{\circ} \mathrm{C}\right)$ between $\mathrm{TSV}$ and the variation rate of skin temperature after subject changed their activity from walking to sitting, and Hypothesis Testing of the relation can be seen in Table VI. However, poor correlation was found between TSV and the variation rate of skin temperature after subject changed their activity from sitting to walking. 
TABLE VI: ANOVA.

\begin{tabular}{|c|c|c|c|c|c|c|}
\hline Model & & Sum of squares & $\overline{\mathrm{df}}$ & Mean Square & $\mathrm{F}$ & Sig. \\
\hline \multirow[t]{3}{*}{ Slightly cool } & Regression & 0.345 & 1 & 0.350 & 34.886 & 0.000 \\
\hline & Residual & 0.080 & 8 & 0.010 & & \\
\hline & Total & 0.430 & 9 & & & \\
\hline \multirow[t]{3}{*}{ neutral } & Regression & 0.648 & 1 & 0.648 & 11.926 & 0.0086 \\
\hline & Residual & 0.435 & 8 & 0.054 & & \\
\hline & Total & 1.082 & 9 & & & \\
\hline
\end{tabular}

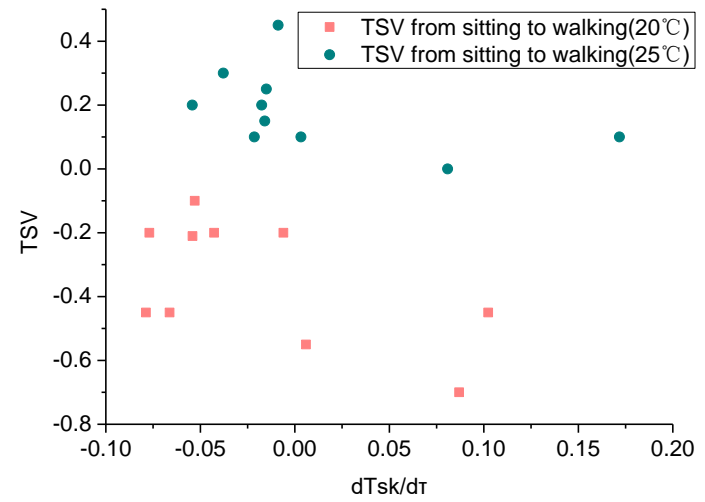

(a) From sitting to walking

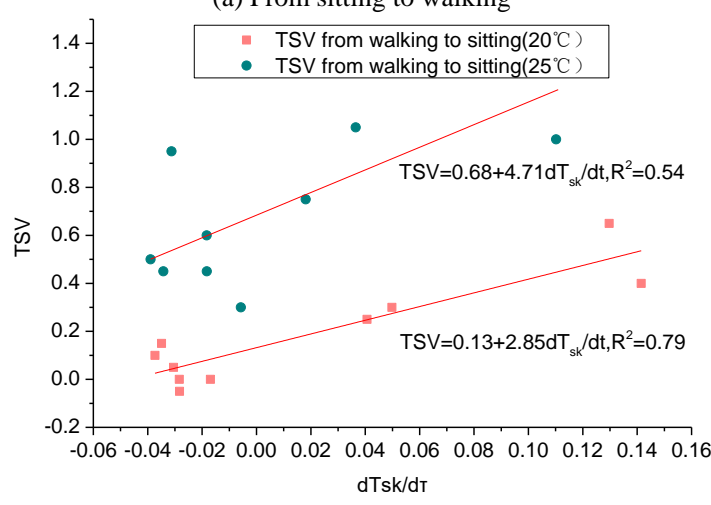

(b) From walking to sitting

Fig. 9. Thermal sensation as a function of variation rate of skin temperature for the first $5 \mathrm{~min}$ after activity level changed in both cases.

\section{CONCLUSIONS}

1) The TSV in neutral condition was more sensitive to and changed faster with down-steps activities, which was in accordance with the variation of TSV in response to warm-to-cold changes. While in the cold condition, the TSV was more sensitive to up-steps activities.

2) A single index, skin temperature is not sufficient to predict thermal sensation of people occupying in dynamic activity, while in the first five minutes after people change their activity from sitting to walking, variation rate of skin temperature is much better than skin temperature to predict thermal sensation. The overall thermal sensation correlates significantly with the variation rate of temperature with time in approximately linear relationship in the first few minutes when subjects change their activity from walking to sitting. When subjects change their activity from sitting to walking, however, the linear relationship between thermal sensation and variation rate of skin temperature disappears. More parameters need to be measured to find the index predicting human thermal sensation.

\section{REFERENCES}

[1] P. O. Fanger, Thermal Comfort: Analysis and Applications in Environmental Engineering, Copenhagen: Danish Technology Press, 1970.

[2] Ergonomics of the thermal environment - Analytical determination and interpretation of thermal comfort using calculation of the PMV and PPD indices and local thermal comfort criteria, Geneva, ISO7730-2005.

[3] Thermal Environment Conditions for Human Occupancy, ANSI/ASHRAE Standard 55.

[4] Evaluation Standard for Indoor Thermal Environments in Civil Buildings, Beijing, China, GB50785- 2012.

[5] Y. Juan, "Studies on the effects of physiological acclimatization on thermal response of people accustomed to different indoor thermal environment," Donghua University, Shanghai, China 2011.

[6] W.-W. Liu, "Study on objective evaluation index of human thermal comfort," Shanghai Jiao Tong University, China, 2008.

[7] M.-L. Tan, "Effects of air humidity and velocity on human thermal sensation in summer," Chongqing University, China, 2012.

[8] B. Olesen, ASHRAE Journal, August: Guidelines for Comfort, pp. 41-46, 2000.

[9] A. P. Gagge, J. A. Stolwijk, and B. Saltin, "Comfort and thermal sensations and associated physiological responses during exercise at various ambient temperatures," Environmental Research, vol. 2, p. 209, Feb. 1969.

[10] R. Nielsen and T. L. Endrusick "Sensations of temperature and humidity during alternative work rest and the influence of underwear knit structure," Ergonomics, vol. 33, pp. 221-234, Feb. 1990.

[11] T. Goto et al., "Thermal sensation and thermos-physiological responses to metabolic step-changes," International Journal of Biometeorology, vol. 50, pp. 323-332, May, 2006.

[12] R. J. Ring J. W. Fanger, "Thermal sensations resulting from sudden ambient temperature changes," Indoor Air, vol. 3, pp. 181-192, March, 1993.

[13] H. Zhang et al., "Thermal sensation and comfort models for non-uniform and transient environments, part III: Whole-body sensation and comfort," Building and Environment, vol. 45, pp 399-410, Feb. 2010.

Diyi Tan is a master student in Chongqing University whose research field is human thermal comfort and indoor ventilation methods.

Hong Liu is a professor in Chongqing University, who is the deputy director of Joint International Research Laboratory of Green Buildings and Built Environments, Ministry of Education, P.R. China. Hong Liu is one member of ASHRAE. Hong Liu's Research field is indoor thermal environment.

Yuxin Wu is a master student in Chongqing University whose research field is human adaptive behavior to achieve thermal comfort and thermal environment in aircraft cabin. 